\title{
Pflanzen der High Society
}

\author{
Hilke Steinecke
}

Im Winter 2011 gab es wieder eine neue Reihe abendlicher Führungen durch das dämmerige Tropicarium. Zwischen Januar und März traf sich an insgesamt 14 Terminen ein bis zwei Mal pro Woche eine Gruppe, um die „Lieblingspflanzen der High Society“ zu erkunden. Auf dem Progamm standen Pflanzen, die nach Kaisern, Königen oder anderen Prominenten benannt worden sind. Zudem gab es viele Geschichten um beliebte Gewächse und Blumen bekannter Persönlichkeiten.

Wie auch schon in den Vorjahren traf sich die Gruppe zunächst im mit Kerzen beleuchteten Eingangsoktogon des Tropicariums. Dort gab es eine gemeinsame Einführung, bevor die Gruppe geteilt wurde und die eine Hälfte von der Wüste in die Mangrove ging, während die andere in entgegengesetzter Richtung wanderte. Die entsprechenden Stationen waren durch brennende Teelichter gekennzeichnet. Der ursprüngliche Pflanzenbestand des Palmengartens geht auf eine wertvolle Pflanzensammlung eines Adeligen zurück. Im Jahre 1866 wurden Frankfurt und Hessen-Nassau preußisch, als Folge musste Herzog Adolph von Nassau seine Residenz in Wiesbaden-Biebrich aufgeben. Dort befand sich seine große Sammlung exotischer Pflanzen, darunter auch Kamelien, die nun zum Verkauf angeboten wurde. Diese Sammlung wurde Grundstock für die Pflanzenkollektion des kurz darauf gegründeten Palmengartens. Zum Einstieg wurden einige Pflanzen mit „edlen Namen“ vorgestellt, von der Kaiserkrone über die Königspalme, den Rittersporn, das Edelfräulein (Klatschmohn) bis hin zur Edelmannsblume (Margerite). Hervorgehoben wurde auch die Ruhmeskrone (Gloriosa rothschildiana). Diese attraktive lilienähnliche Kletterpflanze wurde nach dem zweiten Baron Rothschild (1668-1937) benannt.

Abb. 1: Kaiserkrone (Fritillaria imperialis) vor dem Blütenhaus.

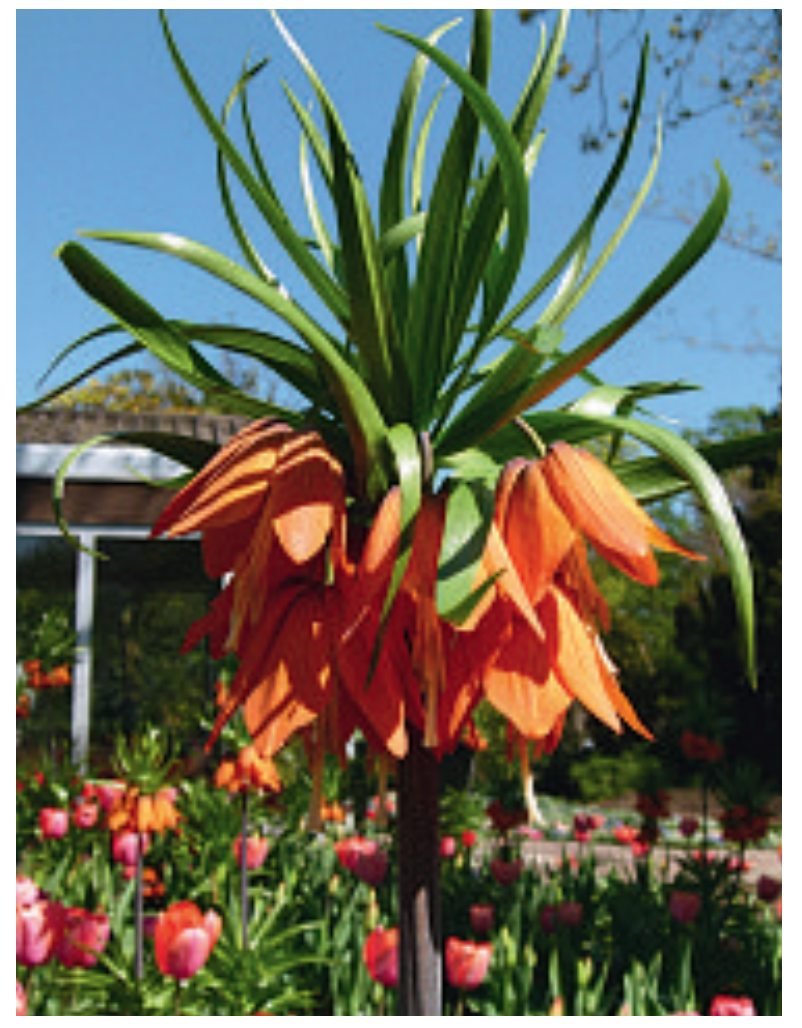

Mitglieder der Bankiersfamilie Rothschild waren sehr an Gärten und Pflanzen interessiert und finanzierten Sammelreisen von Pflanzenjägern. Die Marmorvasen im Rosengarten wurden von Freifrau Meyer Carl von RothSCHILD im April 1892 dem Palmengarten gestiftet.

Ein kurzes Stück ging es dann durch die Kälte in das Mangroven-Haus, dementsprechend beschlugen dort die Brillengläser. Deshalb wurde zunächst auch der Tastsinn angesprochen. In einem goldenen Säckchen sollten Erbsen erfühlt werden. Anknüpfungspunkt war das Märchen von der Prinzessin auf der Erbse. Im Märchen wurde die Prinzessin von einer kleinen Erbse grün und blau gedrückt. Was wäre allerdings, wenn das Märchen in den Tropen spielen würde, wo viel größere Hülsenfrüchte und dementsprechend dicke Samen zur Verfügung stehen? Die große Frucht einer Meerbohne (Entada phaseoloides) beeindruckte die 

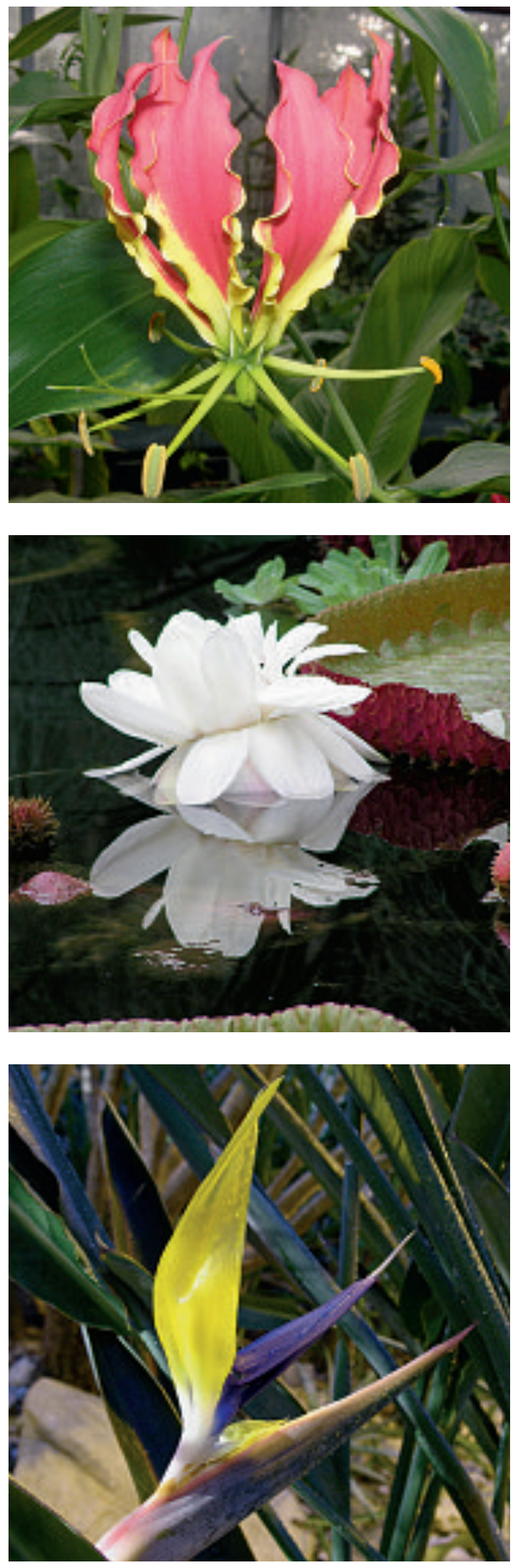

Gruppe sehr. Im Mangroven-Haus gab es noch weitere Stationen. Wappenpflanzen wie die Französische Lilie, die eigentlich eine Iris ist, sowie die schottische Distel wurden vorgestellt. Nach KarL dem Großen wurde die Silberdistel (Carlina acaulis) benannt. Es wird erzählt, dass seine Soldaten während eines Feldzuges schwer erkrankten. Der Erzengel Gabriel schickte ihm einen Pfeil, mit dem er auf eine Pflanze zielen sollte, mit derern Hilfe die Männer gerettet werden könnten. Er traf die Silberdistel, die so starke Heilkraft besaß, dass die Soldaten gesund wurden.

Wasserpflanzen begeisterten nicht nur unsere Besucherinnen und Besucher, sondern faszinierten auch Adelige. Zu Beginn des 20. Jh. sah eine thailändische Prinzessin im Botanischen Garten von Bogor/Indonesien eine Wasserhyazinthe (Eichhornia crassipes), die sie nach Thailand in ihren Palastgarten mitnahm. Damals war noch nicht bekannt, dass die aus Südamerika stammende Wasserpflanze sich mangels natürlicher Feinde rasant vermehren kann und z.B. Staudämme zuwuchert sowie Turbinen und Schiffsschrauben verstopft. Schon wenige Jahre nach ihrer Einführung galt die Wasserhyazinthe in Thailand als ein invasives „Unkraut". Die Pflanze ist heute weltweit in den Tropen verbreitet, über ihre oft riesigen Bestände wird man kaum Herr. Interessant aber, welche Nutzungsmöglichkeiten es gibt, wie z. B. die Verarbeitung zu Körben und Möbeln.

Vorbei an dem afrikanischen und nach NAPOLEON benannten Strauch Napoleonaea vogelii ging es in den Nebelwald. Da hier gerade Kamelien blühten, wurde das Thema Orangerien und Knopflochblumen aufgegriffen. Passend dazu hatten sich die beiden Führer auch mit einer Kamelie im Knopfloch dekoriert. Berühmte Knopflochblumenträger waren z. B. OsKar Wilde, Humphrey Bogart, Charles BauDELAIRE oder in heutiger Zeit auch Prinz

Abb. 2 (oben): Ruhmeskrone (Gloriosa rothschildiana).

Abb. 3 (Mitte): Riesen-Seerose (Victoria amazonica).

Abb. 4 (unten): Paradiesvogelblume (Strelitzia reginae 'Mandelas Gold'). 
Charles. Als Knopflochblumen wurden außer Kamelien häufig auch Chrysanthemen und Orchideen verwendet. Ergänzend zur Napoleonaea wurde Napoleons Gattin Josephine vorgestellt. Sie stammte aus der Karibik von der französischen Insel Martinique und ist deshalb schon in ihrer Jugend mit prächtigen Blumen und Gärten vertraut gewesen. Durch ihre erste Ehe gelangte sie nach Frankreich, wo sie später auch Napoleon kennen und lieben lernte. Beide waren große Verehrer der Veilchen, JosePHINEs Brautkleid beispielsweise war mit Veilchen verziert. Um Veilchen gibt es unendlich viele Geschichten, so auch, dass Goethe die Veilchen so sehr liebte. Auf seinen Spaziergängen rund um Weimar hatte er stets Veilchensamen dabei, die er an verschiedenen Stellen ausstreute. Zitruspflanzen, die typischen Gewächse der Orangerien, gab es im Nebelwald-Haus nicht zu sehen, dafür war hier aber eine kleine flüssige Stärkung auf Orangenbasis versteckt.

Manch einer hat sicherlich schon probiert, eine Ananaspflanze aus dem Schopf einer Frucht heranzuziehen. Oft missglückt das, da das Klima in der Wohnung nicht tropischfeucht genug ist. Im Palmengarten ist das kein Problem, im Bromelienhaus war im Winter eine junge heranwachsende Ananasfrucht zu bewundern. Ananas gilt als die Königin der Früchte, im 19. Jh. war die teure Frucht ein Statussymbol. Trotz des oft nasskalten Wetters in England gelang es der Queen Victoria, ihren Gästen regelmäßig frische Ananasfrüchte anzubieten. Die entsprechende Wärme konnte in den Gewächshäusern mit Hilfe von Mist erzeugt werden. Victorianische Ananasgewächshäuser, die mit Mist beheizt werden, wurden in den verlorenen Gärten von Heligan (Lost Gardens of Heligan) in Cornwall rekonstruiert. Für jeden gab es anschließend ein Häppchen frische Ananas.

Die Königinnen der Blumen sind Rosen oder auch Orchideen. Eine der wertvollsten Orchideen ist die in Mittel- und Südamerika heimische Cattleya. Arten dieser Gattung entwickeln

Abb. 5: Üppig blühende Königin der Nacht (Selenicereus grandiflorus) in der botanischen Sammlung.

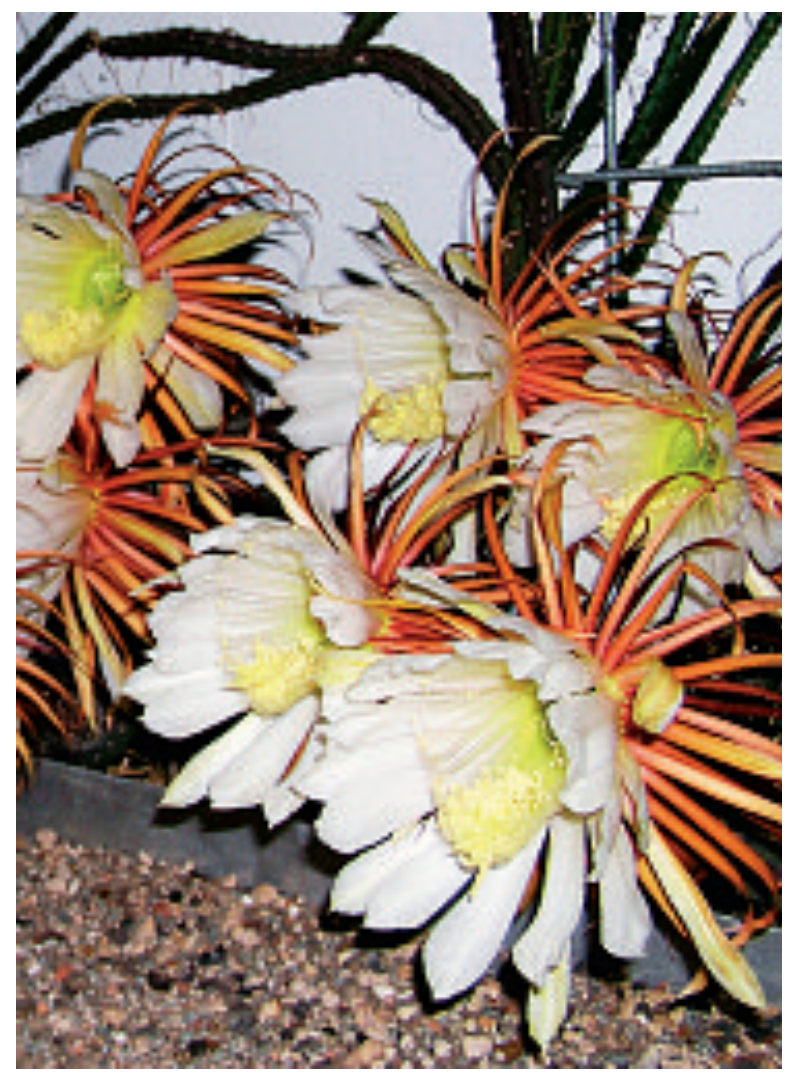

oft sehr große, farbige Blüten mit einem intensiven Duft. Cattleyen wurden in der Literatur aufgegriffen, so auch in dem Roman „Eine Liebe von Swann" von Marcel Proust. In dem Roman, der um 1900 in Paris spielt, lässt sich der reiche Kunstliebhaber und Schürzenjäger Charles Swann mit der koketten Halbweltdame Odette de Crécy ein. In ihrer Beziehung wissen beide, dass es intim wird, wenn sie die Cattleya zurechtrücken gehen.

Auch heimische Pflanzen sind nicht weniger spannend. So gilt die Kornblume als die Lieblingspflanze der Preußenkönigin Luise. Ihr Sohn, der spätere Kaiser Wilhem I., erinnerte sich gern an die Situation 1806 während der Flucht nach Ostpreußen. Als die Familie eine Wagenpanne hatte, flocht die Mutter ihren Kindern einen Kranz aus Kornblumen. Die stängellose Primel ist Symbolpflanze des Primrose Day (Primel-Tag) am 19. April. Die Primel war Lieblingsblume des britischen Romanschriftstellers und Premierministers Benjamin Disraeli. Der Primel-Tag wurde bis zum ersten Weltkrieg zu DisRaelis Ehren begangen und sein Grab mit Primeln bepflanzt. 


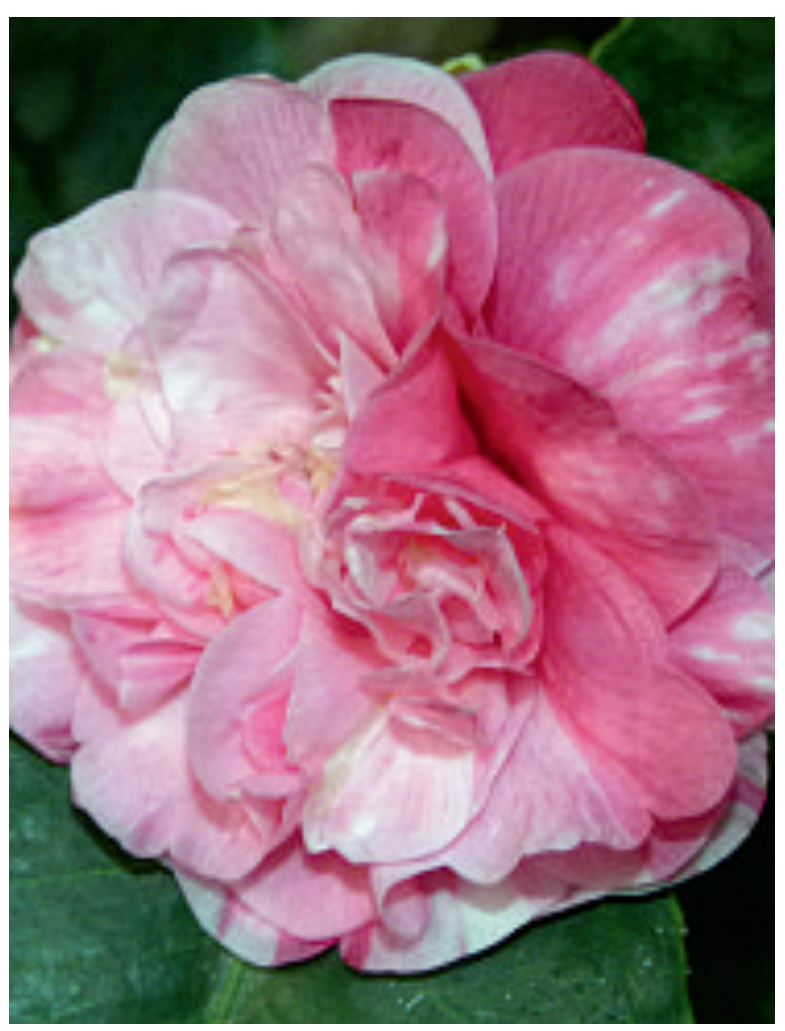

Im Trockenwald-Haus gab es eine Station am Affenbrotbaum oder Baobab (Adansonia digitata). Es wurden die unterschiedlich geformten, bis kopfgroßen Früchte dieser Art gezeigt und verschiedene Baobab-Produke, die auch bei uns erhältlich sind (z. B. Fußbalsam aus dem Samenfett) vorgestellt. Der Baobab wurde auch durch das Märchen vom Kleinen Prinzen von Antoine de Saint-Exupéry bekannt. In dieser Geschichte tritt das Problem auf, dass die Samen schnell keimen und die Bäume so rasch wachsen, dass sie den ganzen Planeten überwuchern würden, wenn sie nicht regelmäßig gejätet würden. In der Natur ist der Baobab aber keinesfalls eine so schnell und invasiv wachsende Art. Zusammen mit einem kleinen selbst gebastelten kleinen Prinzen für den Blumentopf und einer Kopie des entsprechenden Kapitels aus dem Märchen ging es weiter zur Rosenstation.

Viel Interessantes gibt es über Rosen zu berichten, z. B. dass sie von Kaiserin Josephine in ihrem Garten von Malmaison gesammelt und kultiviert wurden, dass sie ihr Hofmaler REDOUTÉ gezeichnet hat oder dass in England Rosenkriege zwischen den Häusern York und Lan- caster geführt wurden. Rosen haben auch in Hessen große Bedeutung, gibt es doch die Rosenzüchter in Steinfurt, die Rosenhöhe in Darmstadt und unseren schönen Rosengarten im Palmengarten. Um für Hessen zu werben, wurde ein Parfüm namens Hessenduft entwickelt, das den Duft von Äpfeln und Rosen enthält. Gern wurde an dem Duftfläschchen geschnuppert.

Im Halbwüsten-Haus erklang MozarTs Arie von der Königin der Nacht neben dem Kletterkaktus Königin der Nacht (Selenicereus grandiflorus). In Sommernächten öffnet er für nur wenige Stunden seine großen weißen Blüten, die von Fledermäusen bestäubt werden. Kakteenliebhaberinnen gab es auch in der High Society. Man erzält, dass Marie Antoinette, zum Tode verurteilte französische Kaiserin, in ihrem Gefängnis auch einen Kaktus hielt. Als er das erste Mal blühte, war es ihr sehr wichtig, ihren Hofmaler, ebenfalls Redouté, zu rufen, damit er dieses Blühereignis festhalte.

Zum Abschluss der Führung wurde die Nebelwüste besichtigt. Es ist immer ein sehr schöner Kontrast, aus den dämmrigen Gewächshäusern in die hell erleuchtete Nebelwüste einzutreten. Blickfang waren hier mehrere Exemplare der Pradiesvogelblume (Strelitzia reginae). Die Paradiesvogelblume stammt aus den Küstenwäldern der östlichen Kap-Provinz und wird heute in großen Mengen als Schnittblume angebaut. Entdeckt und nach Europa gebracht wurde sie 1773 von dem Pflanzenjäger Francis Masson. Der Gattungsname ehrt Charlotte Sophia von Mecklenburg-Strelitz, die spätere Ehefrau des britischen Königs GeORG III. Da sie sich sehr für Pflanzen interessierte und Förderin der Königlichen Gärten in Kew war, wurde sie auch Queen of Botany genannt. Hier gezeigt wurde die seltene Sorte 'Mandelas Gold' mit gelben statt orangen Blüten.

Durch die Halbwüste, vorbei an Agave victoria-reginae, ging es dann schließlich nach etwa zwei Stunden wieder zur Weltkugel, dem Ausgangs- und Endpunkt der Führung.

Abb. 6: Kamelie (Camellia japonica 'Prince Albert') in der Kamelienausstellung 2011. 